\title{
Nonlinear Hybrid Controller for a Quadrotor Based on Sliding Mode and Backstepping
}

\author{
Zhang Lian Chuan, Kil To Chong \\ Department of Electronic Engineering, Chonbuk National University
}

\begin{tabular}{l}
\hline \hline Article Info \\
\hline Article history: \\
Received Apr 12, 2015 \\
Revised Jul 26, 2015 \\
Accepted Aug 10, 2015 \\
\hline Keyword: \\
Backsteping \\
Nonlinear Hybrid Control \\
Quadrotor \\
Sliding Mode
\end{tabular}

\begin{abstract}
In this paper, one nonlinear hybrid controller, based on backstepping and sliding mode, was developed and applied to a quadrotor for waypoint navigation application. After dynamics modeling, the whole quadrotor dynamics system could be divided into two subsystems: rotational system and translational system. Backstepping control law was derived for attitude control whereas sliding mode control law was developed for position control. By using Lyapunov theory and satisfying sliding stable rules, the convergence of system could be guaranteed. A nonlinear equation was proposed to solve the under-actuated problem. To validate the effectiveness of proposed nonlinear hybrid controller, waypoint navigation simulation was performed on the nonlinear hybrid controller. Results showed that the nonlinear hybrid controller finished waypoint navigation successfully.
\end{abstract}

Copyright (C) 201x Institute of Advanced Engineering and Science. All rights reserved.

Corresponding Author:

Kil To Chong,

Department of Electronic Engineering,

Chonbuk National University,

567 Baekje-daero, Deokjin-gu, Jeonju-si, Jeollabuk-do 561-756, South Korea.

Email: kitchong@jbnu.ac.kr

\section{INTRODUCTION}

Fixed wing unmanned aerial vehicles (UAV) have been widely utilized by military and civilian applications such as reconnaissance and rescue. Comparing to fixed wing UAVs, recently, quadrotors are becoming really popular among researchers due to its low cost, easy maintenance and vertical taking off and landing capabilities. Translational and rotational motions of quadrotors can be obtained by changing angular speeds of four electric motors [1]. One quadrotor is a typical under-actuated and coupling system, so there are many technical and theoretical challenges on the dynamics modeling and design of controller. In terms of dynamics modeling, academic research can be divided into two directions: linear dynamic models and nonlinear dynamic models. The latter one contains several nonlinear factors such as attack of angles and blade flapping. Small angles approximation is the most common linearization of linear dynamic models after ignoring nonlinear factors. In terms of quadrotor controllers, it is universal between indoor and outdoor quadrotors, which also have two main directions: linear controllers and nonlinear controllers. Proportional integral derivative (PID) and linear quadratic regulator (LQR) [2] controller are representatives of linear controllers. Linear controllers, especially PID controller, can get good results in both simulations and experiments with small disturbances and operation angles. Besides that, most commercial quadrotors' controller are based on PID algorithm and show good stability and manipulability. Whereas linear controllers fail to finish aggressive maneuver experiments since linearization dynamics model is no longer suitable for big attack angles and high speed situation. To overcoming limitations and drawbacks of linear controllers, researchers begin to focus on nonlinear controllers. Feedback linearization, model predictive control, dynamics inversion, adaptive control, sliding mode, nested saturation-based nonlinear controller, backstepping, integral backstepping and quaternion-based nonlinear controller have been paid much attention [3-7]. 
Although many nonlinear controller have been developed for quadrotors control, backstepping and sliding mode show better performance on solving under-actuated and coupling problem of the quadrotor control. Based on that reason, some papers try to combine sliding mode and backstepping scheme together. Modirrousta's paper [8] combines integral backstepping control with adaptive terminal sliding mode to control the attitude of one quadrotor, which effectively solves chattering problem of sliding mode control. T. Madani's paper [9] derives a sliding mode controller based on backstepping control, which make it easier to choose sliding surface for sliding mode control and ensure Lyapunov stability. His another paper [10] treats sliding mode as an observer of velocities and external disturbances, which can compensate control inputs. $\mathrm{H}$. Bouadi's paper [11] exploits backstepping approach to get sliding mode control law and takes the high order non-holonomic constraints into account. Being stable is critical for one quad rotor's control, therefore rotational system should be with fast dynamics whereas translational system is suitable with slow dynamics. Because sliding mode control laws do not depend on system parameters and can be more robust, we choose sliding mode as rotational system controller while backstepping is adopted as translational system controller.

The main contribution of this paper is adopting nonlinear quadrotor dynamics model to develop a new hybrid controller based on backstepping and sliding mode method. Firstly, Newton-Euler approach is utilized to obtain nonlinear dynamics model. Based on the dynamic model, the whole system can be divided into two subsystems: translational and rotational system. Backstepping controller is developed for translational system while sliding mode controller is proposed for rotational system. Depending on translational motion equations of dynamics model, one nonlinear equation is derived to solve under-actuated problem and build connections between these two subsystems. Finally, the new hybrid controller has been obtained. Waypoint navigation simulation is performed on the hybrid controller to validate the performance of the nonlinear hybrid controller. Simulation results show successful performance of the proposed controller. In the next section quadrotor dynamics modeling is discussed. Then in Section III backstepping and sliding mode control law is developed to get the nonlinear hybrid controller. Simulation results are described in Section IV. Finally, in Section V, conclusion and discussion are presented.

\section{DYNAMICS MODELING}

Quadrotor dynamics model is usually obtained by two different approaches: Euler-Lagrange and Newton-Euler equations, and these two approaches can get same motion equations. There are many nonlinear factors of complete dynamics of a quadrotor such as free-stream velocity, blade flapping and gyroscopic effect. It would be very complicated even not feasible for the purpose of control if one dynamics model considers all nonlinear effect factors. Therefore, this paper builds a simplified dynamics model which retains main features and ignores some nonlinear effect factors such as free-stream velocity and blade flapping, which are easily observed in aggressive motions of large quadrotors [12]. In this paper, the dynamics model will be derived based on Newton-Euler equations[13] under the assumption that the center of mass coincides with the body fixed frame. Based on Newton-Euler formalism, the dynamics of a rigid body in body frame $B$ can be described as:

$$
\left[\begin{array}{cc}
m_{3} & 0 \\
0 & J
\end{array}\right]\left[\begin{array}{c}
\dot{V} \\
\dot{\Omega}
\end{array}\right]+\left[\begin{array}{c}
\Omega \times m V \\
\Omega \times J \Omega
\end{array}\right]=\left[\begin{array}{c}
F_{\text {ext }} \\
\tau
\end{array}\right],
$$

Where $I$ is the identity matrix; $V=(u, v, w)$ and $\Omega=(p, q, r)$ represent, respectively, the linear and angular velocities in the body-fixed frame; $F_{e x t}$ and $\tau$ are the total external force and torque, respectively; and $J$ is the moment of inertia which is given by:

$$
\boldsymbol{J}=\left[\begin{array}{ccc}
I_{x} & 0 & 0 \\
0 & I_{y} & 0 \\
0 & 0 & I_{z}
\end{array}\right]
$$

Using Euler angles parameterization, one can use a rotation matrix $R$ to express translational dynamics in inertial frame, where $R$ is defined as follows:

$$
R=\left[\begin{array}{ccc}
c \theta c \psi & s \phi s \theta c \psi-c \phi s \psi & c \phi s \theta c \psi+s \phi s \psi \\
c \theta s \psi & s \phi s \theta s \psi+c \phi c \psi & c \phi s \theta s \psi-s \phi c \psi \\
-s \theta & s \phi c \theta & c \phi c \theta
\end{array}\right]
$$


Where $\eta=(\phi, \theta, \psi)$ denotes three Euler angles roll, pitch, and yaw, respectively; $S$ and $C$ are abbreviations for sin and cos function. By considering this transformation, the translational dynamics of inertial frame are computed as follows:

$$
m \dot{V}=R F_{B}-\left[\begin{array}{lll}
0 & 0 & m g
\end{array}\right]^{T},
$$

Where $V=(\dot{x}, \dot{y}, \dot{z})$ the rotorcraft velocity in inertial frame and $g$ is the gravitational acceleration and $F_{B}$ is the total force excluding the gravity force.

The original Newton-Euler equations are derived based on rigid body which does not consider internal dynamics. Since electric motor's gyroscopic effect is obvious, this paper adds gyroscopic effect term to Newton-Euler equations. The new dynamics equations for rotational motion can be rewritten as:

$$
J \dot{\Omega}+\Omega \times J \Omega+\Omega \times\left[\begin{array}{lll}
0 & 0 & J_{r} \Omega_{r}
\end{array}\right]^{T}=\tau,
$$

Where $J_{r}$ is motor's moment of inertia and $\Omega_{r}$ is residual angular speed of four motors $\left(w_{1}, w_{2}, w_{3}, w_{4}\right)$, which can be expressed as:

$$
\Omega_{r}=w_{1}-w_{2}+w_{3}-w_{4}
$$

To transform attitude dynamics in body-fixed fame into inertia frame, we need the kinematic relation between $\Omega$ and $\dot{\eta}$ :

$$
\dot{\eta}=R_{r} \Omega,
$$

Where the Euler matrix $R_{r}$ is given by

$$
R_{r}=\left[\begin{array}{ccc}
1 & \sin (\phi) \tan (\theta) & \cos (\phi) \tan (\theta) \\
0 & \cos (\phi) & -\sin (\phi) \\
0 & \sin (\phi) \sec (\theta) & \cos (\phi) \sec (\theta)
\end{array}\right] .
$$

We can make assumption around hover state and small angles where $\psi \approx 0, \theta \approx 0$. Based on that assumption, this transformation matrix can be simplified to an identity matrix, which means that actually no changes are made on rotational dynamics. Depending on this approximation, the rotational dynamics of inertial frame can be calculated as follows:

$$
J \ddot{\eta}+\dot{\eta} \times J \dot{\eta}+\Omega \times\left[\begin{array}{lll}
0 & 0 & J_{r} \Omega_{r}
\end{array}\right]^{T}=\tau .
$$

A quadrotor is an under-actuated system with 6 degree of freedom and 4 control inputs, which are the total thrust $U_{1}$ and the torques $\left(U_{2}, U_{3}, U_{4}\right)$. Hence the force and torque vectors in equation (4) and equation (5) can be expressed as $F_{B}=\left[\begin{array}{lll}0 & 0 & U_{1}\end{array}\right]^{T}$ and $\tau=\left[\begin{array}{llll}U_{2} & U_{3} & U_{4}\end{array}\right]^{T}$, respectively. Under the assumption that thrusts are proportional to the square of propeller's speed, the relationship between control inputs $\left(U_{1}, U_{2}, U_{3}, U_{4}\right)$ and rotors' speed $\left(w_{1}, w_{2}, w_{3}, w_{4}\right)$ can be given by

$$
\left[\begin{array}{l}
U_{1} \\
U_{2} \\
U_{3} \\
U_{4}
\end{array}\right]=\left[\begin{array}{cccc}
K_{f} & K_{f} & K_{f} & K_{f} \\
0 & -K_{f} & 0 & K_{f} \\
-K_{f} & 0 & K_{f} & 0 \\
K_{m} & -K_{m} & K_{m} & -K_{m}
\end{array}\right]\left[\begin{array}{c}
w_{1} \\
w_{2} \\
w_{3} \\
w_{4}
\end{array}\right],
$$

Where $K_{f}$ and $K_{m}$ are the aerodynamic force and moment constants respectively. Recalling equation (4) and equation (8), the nonlinear model of a quadrotor can be expressed in the following form, where $l$ denotes the distance between rotors' center and the center of mass: 


$$
\left\{\begin{array}{l}
\ddot{\phi}=\dot{\theta} \dot{\psi}\left(\frac{I_{y}-I_{z}}{I_{x}}\right)-\frac{J_{r}}{I_{x}} \dot{\theta} \Omega+\frac{l}{I_{x}} U_{2} \\
\ddot{\theta}=\dot{\phi} \dot{\psi}\left(\frac{I_{z}-I_{x}}{I_{y}}\right)+\frac{J_{x}}{I_{y}} \dot{\phi} \Omega+\frac{l}{I_{y}} U_{3} \\
\ddot{\psi}=\dot{\psi} \dot{\theta}\left(\frac{I_{x}-I_{y}}{I_{z}}\right)+\frac{l}{I_{z}} U_{4} \\
\ddot{z}=-g+(\cos \phi \cos \theta) \frac{1}{m} U_{1} \\
\ddot{x}=(\cos \phi \sin \theta \cos \psi+\sin \phi \sin \psi) \frac{1}{m} U_{1} \\
\ddot{y}=(\cos \phi \sin \theta \sin \psi-\sin \phi \sin \psi) \frac{1}{m} U_{1}
\end{array}\right.
$$

The dynamics model derived by this section is suitable for mini-quadrotor with small propellers and low flying speed. For a more accurate dynamics for large quadrotors model which considers blade flapping, big angles of attack, one can refer to G.M. Hoffman and S. Bouabdallah's paper [14, 15].

\section{NONLINEAR HYBRID CONTROLLER DESIGN}

It is obvious that there is no coupling among position control outputs in comparison with attitude control outputs, so we can divide the whole dynamics system into two subsystems: translational and rotational system. Sliding mode controller is proposed for attitude control whereas translational control adopts backstepping controller. Sliding mode is a kind of nonlinear and discontinuous control, which can force the system dynamics slide along sliding surface. The biggest advantages of sliding mode is that system dynamics is independent of controller's parameters because entire dynamics of system is governed by the sliding surface. However, oscillation caused by switching between sliding surface is a drawback of sliding mode control [16]. In terms of backstepping, it is feasible for cascade control, which provides a recursive method to stabilize the origin of a system in strict feedback form [3]. In order to apply sliding mode and backstepping approaches, the equations of dynamics should be rewritten into state space form as follows:

$$
\begin{gathered}
\{\dot{X}=f(X, U) \\
X=\left(x_{1} \cdots x_{12}\right)=(\phi, \dot{\phi}, \theta, \dot{\theta}, \psi, \dot{\psi}, z, \dot{z}, x, \dot{x}, y, \dot{y},)^{T}, \\
x_{2} \\
x_{4} x_{6} a_{1}+x_{4} a_{2} \Omega+b_{1} U_{2} \\
x_{4} \\
x_{2} x_{6} a_{3}+x_{2} a_{4} \Omega+b_{2} U_{3} \\
x_{6} \\
x_{2} x_{4} a_{5}+b_{3} U_{4} \\
x_{8} \\
-g+\left(\cos x_{1} \cos x_{3}\right) \frac{1}{m} U_{1} \\
x_{10} \\
x_{12} \\
\frac{U_{1}}{m}(\cos \phi \sin \theta \cos \psi+\sin \phi \sin \psi) \\
a_{1}=\left(I_{y}-I_{z}\right) / I_{x} \\
a_{2}=-J_{r} / I_{x} \\
a_{3}=\left(I_{z}-I_{x}\right) / I_{y} \\
a_{4}=J_{r} / I_{y} \\
a_{5}=\left(I_{x}-I_{y}\right) / I_{z}
\end{gathered} \mid b_{1}=l / I_{x}=l / I_{y}
$$

\subsection{Sliding Mode Controller Design for Attitude Control}

Sliding mode control involves 2 steps. Firstly, sliding surface should be chosen so that the system exhibits desirable behavior when confined to this manifold. After that one needs to derive reaching law so that the system intersects or stays on the sliding surface. To start our design, roll angle tracking error is defined as $e_{1}=\phi_{\text {ref }}-\phi$ and compute its dynamics as:

$$
\frac{d e_{1}}{d t}=\dot{\phi}_{r e f}-w
$$


One can obtain exponential convergence of the system by choosing the angular velocity $w$ as $w=c_{1} e_{1}+\dot{\phi}_{r e f}$. However, angular velocity $w$ is only a system variable instead of system control inputs [17]. It only can be treat as virtual control input and the desirable behavior for this virtual control input is

$$
w_{r e f}=c_{1} e_{1}+\dot{\phi}_{r e f}
$$

Although $w$ is not one of our control inputs, it can make the roll angle tracking error convergent. So we choose the tracking error of $w$ as our sliding mode surface, which can be defined as:

$$
S_{2}=w-w_{r e f}=w-c_{1} e_{1}-\dot{\phi}_{r e f}=x_{2}-\dot{x}_{1 r e f}-c_{1} e_{1} .
$$

There are three general forms for sliding mode control law: constant rate reaching law, constant and proportional reaching law and power rate reaching law, which can be respectively given by following equations:

$$
\left\{\begin{array}{c}
\dot{s}=-k \operatorname{sgn}(s) \\
\dot{s}=-q s-k \operatorname{sgn}(s) \\
\dot{s}=-k(s)^{\alpha}, 0<\alpha<1
\end{array} .\right.
$$

We choose the second form to obtain sliding mode control law. In order to satisfy $S \dot{S}<0$, the time derivative of was chosen to be sliding mode reaching law.

$$
\begin{aligned}
\dot{S}_{2} & =-q_{2} \operatorname{sign}\left(S_{2}\right)-k_{2} S_{2}=\dot{x}_{2}-\ddot{x}_{1 r e f}-c_{1} \dot{e}_{1} \\
& =x_{4} x_{6} a_{1}+x_{4} a_{2} \Omega+b_{1} U_{2}-\ddot{x}_{1 r e f}-c_{1} \dot{e}_{1}
\end{aligned}
$$

The control law of $U_{2}$ can be synthesized by using equation (13) and quation (16),

$$
U_{2}=\frac{1}{b_{1}}\left(-x_{4} x_{6} a_{1}-x_{4} a_{2} \Omega-c_{1}^{2} e_{1}-q_{2} \operatorname{sign}\left(S_{2}\right)-k_{2} S_{2}\right) .
$$

Where $c_{1}, q_{2}$ and $k_{2}$ are positive constants which determines the convergence speed of reaching law. By following the same steps, we can get other two attitude control laws as:

$$
\begin{aligned}
& \left\{\begin{array}{l}
U_{3}=\frac{1}{b_{2}}\left(-x_{2} x_{6} a_{3}-x_{2} a_{4} \Omega-c_{3}^{2} e_{3}-q_{3} \operatorname{sign}\left(S_{3}\right)-k_{3} S_{3}\right) \\
U_{4}=\frac{1}{b_{3}}\left(-x_{2} x_{4} a_{5}-c_{5}^{2} e_{5}-q_{4} \operatorname{sign}\left(S_{4}\right)-k_{4} S_{4}\right)
\end{array}\right. \\
& \text { with }\left\{\begin{array}{l}
e_{3}=x_{3 r e f}-x_{3} \\
S_{3}=x_{4}-\dot{x}_{3 r e f}-c_{3} e_{3} \\
e_{5}=x_{5 \text { ref }}-x_{5} \\
S_{4}=x_{6}-\dot{x}_{5 r e f}-c_{5} e_{5}
\end{array}\right.
\end{aligned}
$$




\subsection{Backstepping Controller Design for Position Control}

We will take one of position outputs as an example to derive backstepping control law [3]. Firstly, we define the tracking error as

$$
e_{7}=z_{\text {ref }}-z=x_{7 r e f}-x_{7} \text {. }
$$

Considering the Lyapunov function of $e_{7}$ positive definite, we choose the following Lyapunov function for $e_{7}$ :

$$
\begin{aligned}
& V\left(e_{7}\right)=\frac{1}{2} e_{7}^{2} \\
& \dot{V}\left(e_{7}\right)=e_{7}\left(\dot{x}_{7 \text { ref }}-x_{8}\right)
\end{aligned}
$$

We can choose $x_{8}$ as $x_{8}=\dot{x}_{7 \text { ref }}+\alpha_{7} e_{7}$ to stabilize $e_{7}$ but $x_{8}$ is one of control outputs instead of control inputs. So we treat $x_{8}$ as our virtual control input and define its tracking error as:

$$
e_{8}=x_{8}-\dot{x}_{7 r e f}-\alpha_{7} e_{7}
$$

The stabilization of $e_{8}$ can be obtained by augmented Lyapunov function:

$$
\begin{aligned}
& V\left(e_{7}, e_{8}\right)=\frac{1}{2}\left(e_{7}^{2}+e_{8}^{2}\right) \\
& \dot{V}\left(e_{7}, e_{8}\right)=e_{8} \dot{x}_{8}-e_{8}\left(\ddot{x}_{7 r e f}-\alpha_{7} \dot{e}_{7}\right)-e_{7} \dot{e}_{7}
\end{aligned}
$$
be rewritten as:

By using equation (13) and equation (24), the time derivative of augmented Lyapunov function can

$$
\dot{V}\left(e_{7}, e_{8}\right)=e_{8}\left(\frac{U_{1} \cos x_{1} \cos x_{3}}{m}-g\right)-e_{8}\left(\ddot{x}_{7 r e f}-\alpha_{7}\left(e_{8}+\alpha_{7} e_{7}\right)\right)-e_{7} e_{8}-\alpha_{7} e_{7}^{2}
$$

The control input $U_{1}$ has appeared in equation (26). In order to satisfy $\dot{V}\left(e_{7}, e_{8}\right)<0$, the control law $U_{1}$ can be extracted as:

$$
U_{1}=\frac{m}{\cos x_{1} \cos x_{3}}\left(e_{7}+g-\alpha_{7}\left(e_{8}+\alpha_{7} e_{7}\right)-\alpha_{8} e_{8}\right)
$$

The term $\alpha_{8} e_{8}$ is added to stabilize $e_{7}$. And same steps can be followed to obtain control law of $U_{x}$ and $U_{y}$ as:

$$
\begin{aligned}
& U_{x}=\left(m / U_{1}\right)\left(e_{9}-\alpha_{9}\left(e_{10}+\alpha_{9} e_{9}\right)-\alpha_{10} e_{10}\right), \\
& U_{y}=\left(m / U_{1}\right)\left(e_{11}-\alpha_{11}\left(e_{12}+\alpha_{11} e_{11}\right)-\alpha_{12} e_{12}\right) \\
& \left\{\begin{array}{l}
e_{9}=x_{9 d}-x_{9} \\
e_{10}=x_{10}-\dot{x}_{9 d}-\alpha_{9} e_{9} \\
e_{11}=x_{11 d}-x_{11} \\
e_{12}=x_{12}-\dot{x}_{11 d}-\alpha_{12} e_{12}
\end{array} .\right.
\end{aligned}
$$

From the translational motion equations, we can see that desired angles $\left(\phi_{d}, \theta_{d}, \psi_{d}\right)$ are the outputs of position system. Considering $\psi_{d}$ is given by an operator [18], One can use equation (30) to solve underactuated problem and get equation (31). 


$$
\begin{aligned}
& \left\{\begin{array}{l}
U_{z}=-g+(\cos \phi \cos \theta) \frac{1}{m} U_{1} \\
U_{x}=(\cos \phi \sin \theta \cos \psi+\sin \phi \sin \psi) \frac{1}{m} U_{1} \\
U_{y}=(\cos \phi \sin \theta \sin \psi-\sin \phi \sin \psi) \frac{1}{m} U_{1}
\end{array}\right. \\
& \left\{\begin{array}{c}
U_{1}=m \sqrt{\left(U_{x}^{2}+U_{y}^{2}+\left(g+U_{z}\right)^{2}\right)} \\
\phi_{d}=\sin ^{-1}\left(m \frac{U_{x} \sin \psi_{d}-U_{y} \cos \psi_{d}}{U_{1}}\right) \\
\theta_{d}=\tan ^{-1}\left(m \frac{U_{x} \cos \psi_{d}+U_{y} \sin \psi_{d}}{g+U_{1}}\right)
\end{array}\right.
\end{aligned}
$$

With (31), the under-actuated problem is solved and then the control system advocated for the overall system is schematized in figure 1 .

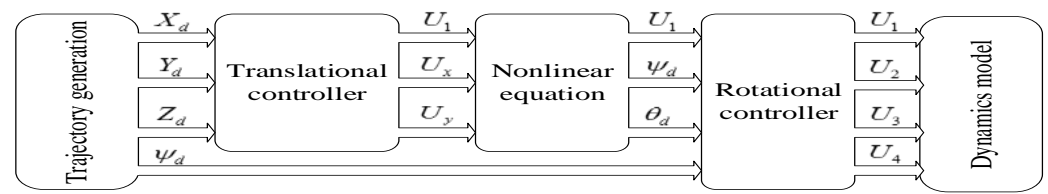

Figure 1. Schematic of nonlinear hybrid controller

\section{SIMULATION RESULTS}

Figure 2 describes waypoint navigation simulation. In this test, the quadrotor is commanded to perform automatic takeoff, waypoint navigation, and stationary flight at $20 \mathrm{~m}$ high, and automatic landing. The fight path is defined by 4 points and the reference trajectories are generated in real time. As figure 2 shows, the simulation trajectory exactly matches desired trajectory and tracking error is almost zero. Figure 3 presents the performance of each axis tracking results while figure 4 shows that attitude tracking errors in radian is small. These results demonstrate that the quadrotor passed successfully through all the points and the performance of nonlinear hybrid controller is good and robust.

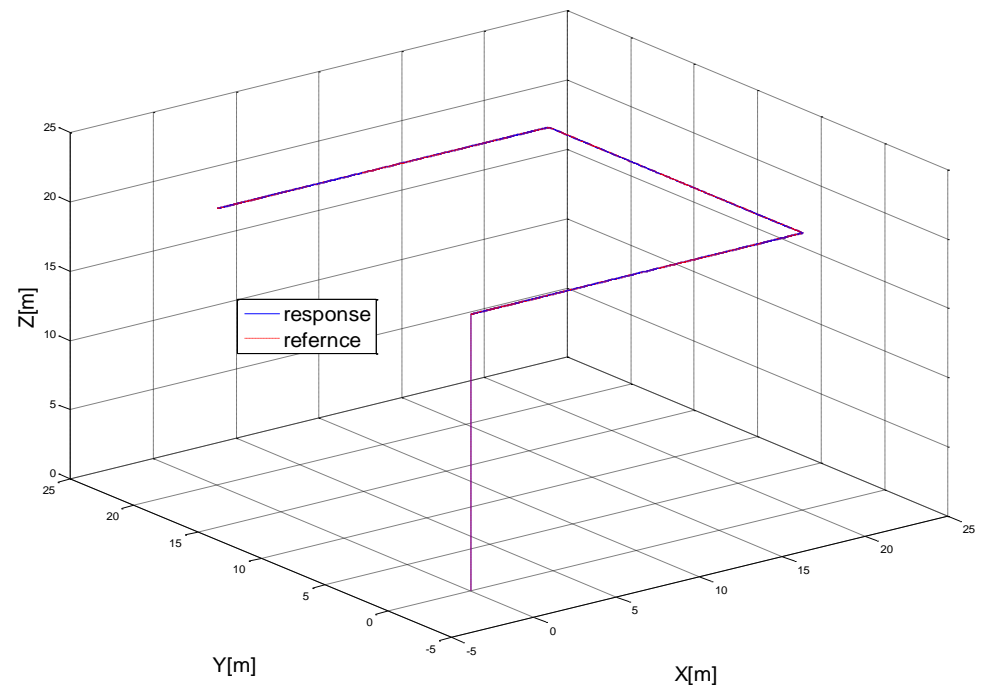

Figure 2. Way point navigation in simulation. 

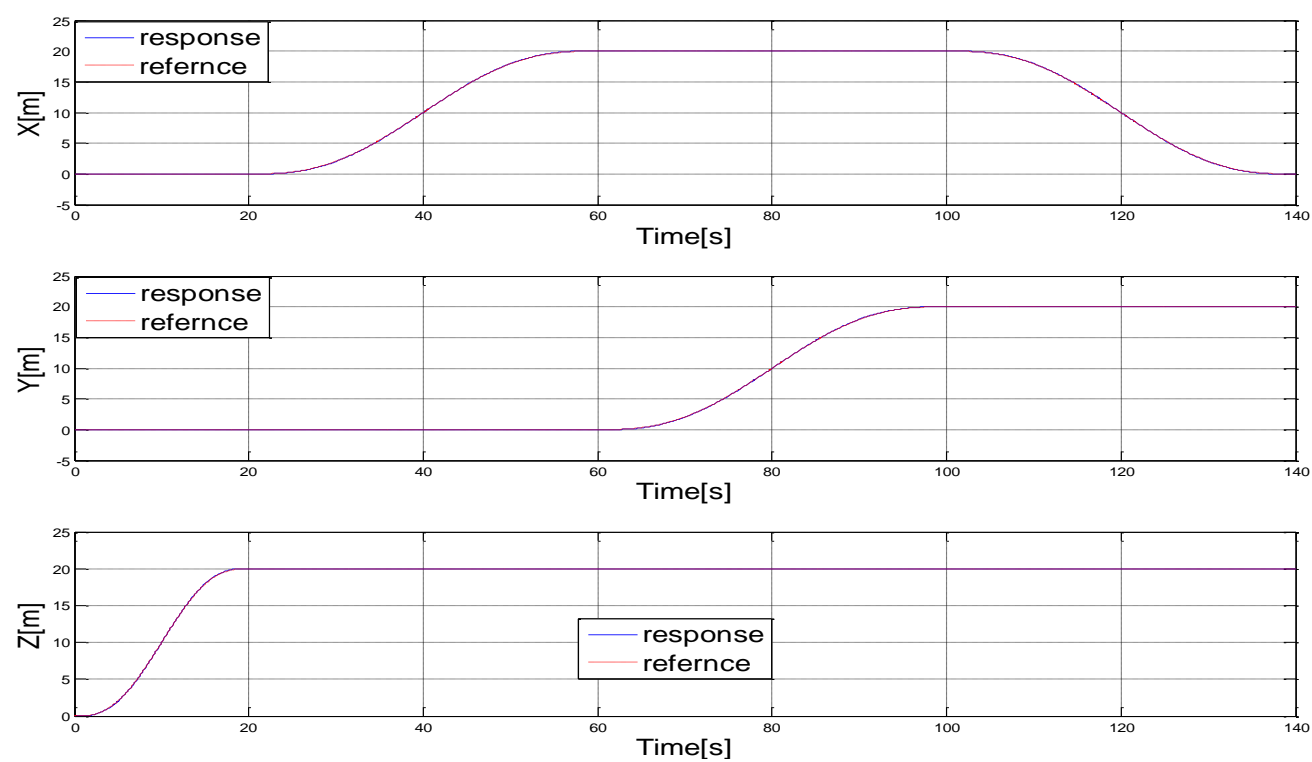

Figure 3. Position outputs of waypoint navigation simulation.

\section{CONCLUSION}

This research dealt with dynamic modeling which considers main features and ignores some nonlinear factors. In this paper, we have described the design of a nonlinear hybrid controller of a quadrotor based on backstepping and sliding mode, which can fulfill waypoint navigation. Indeed, we apply backstepping and sliding mode to translational and rotational control of quadrotor, respectively. The system stability is guaranteed by Lyapunov theory and satisfying sliding mode stabile rules. Simulations have been done to demonstrate the ability of the hybrid controller to provide effective waypoint navigation. From the simulation results, it is concluded that designed hybrid controller shows good and reliable performance and make it possible for practical experiments.

Recently, there are many big breakthroughs about quadrotors' fully automatic flight control and formation control. However, large computation burden makes it difficult to implement complicated algorithms on hardware and short cruise time also confines quadrotors to be applied in practical applications. In the future work, the control system can be improved by considering nonlinear factors caused by high speed flight and aggressive maneuvers.
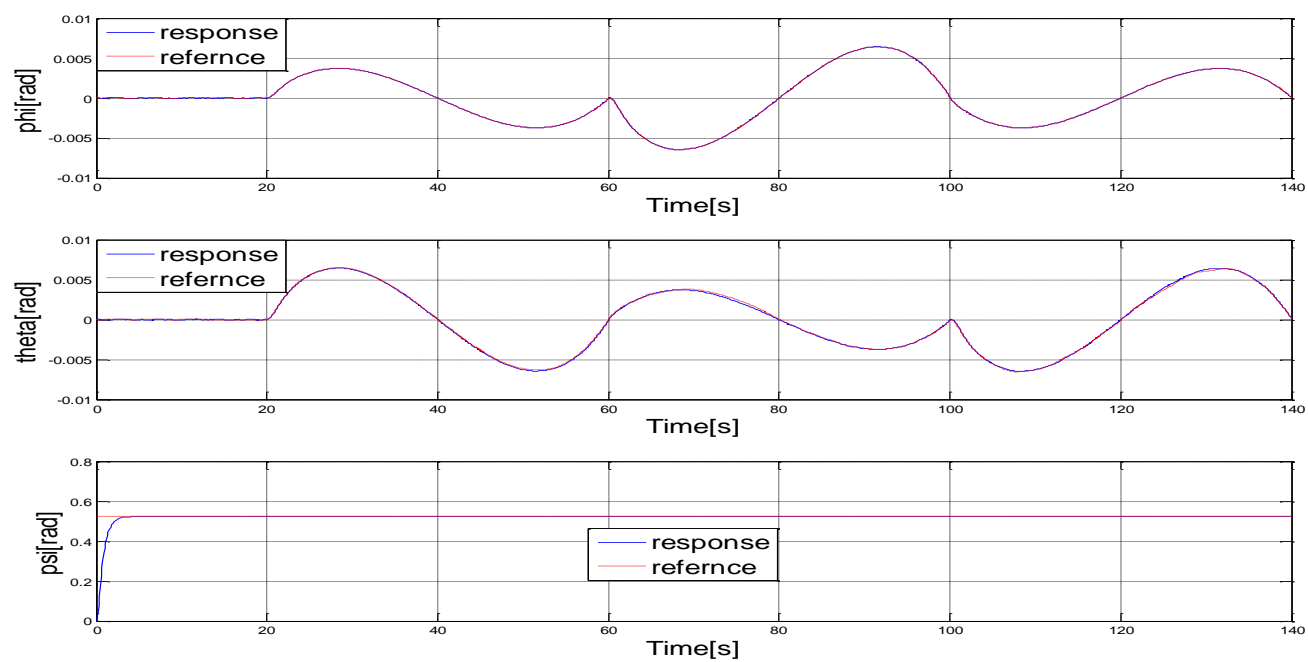


\section{ACKNOWLEDGEMENTS}

Figure 4. Angles outputs of waypoint navigation simulation

This research was supported by Basic Science Research Program through the National Research Foundation of Korea (NRF) funded by the Ministry of Science, ICT \& Future Planning (NRF2013R1A2A2A01068127).

\section{REFERENCES}

[1] W. Wang, K. Nonami, M. Hirata, and O. Miyazawa, "Autonomous control of micro flying robot," Journal of Vibration and Control, vol. 16, pp. 555-570, 2010.

[2] S. Bouabdallah, A. Noth, and R. Siegwart, "PID vs LQ control techniques applied to an indoor micro quadrotor," in Intelligent Robots and Systems, 2004.(IROS 2004). Proceedings. 2004 IEEE/RSJ International Conference on, 2004, pp. 2451-2456.

[3] T. Madani and A. Benallegue, "Backstepping control for a quadrotor helicopter," in Intelligent Robots and Systems, 2006 IEEE/RSJ International Conference on, 2006, pp. 3255-3260.

[4] P. Bouffard, A. Aswani, and C. Tomlin, "Learning-based model predictive control on a quadrotor: Onboard implementation and experimental results," in Robotics and Automation (ICRA), 2012 IEEE International Conference on, 2012, pp. 279-284.

[5] A. Das, K. Subbarao, and F. Lewis, "Dynamic inversion with zero-dynamics stabilisation for quadrotor control," IET control theory \& applications, vol. 3, pp. 303-314, 2009.

[6] T. Lee, M. Leoky, and N. H. McClamroch, "Geometric tracking control of a quadrotor UAV on SE (3)," in Decision and Control (CDC), 2010 49th IEEE Conference on, 2010, pp. 5420-5425.

[7] I. Voos, "Nonlinear control of a quadrotor micro-UAV using feedback-linearization," in Mechatronics, 2009. ICM 2009. IEEE International Conference on, 2009, pp. 1-6.

[8] A. Modirrousta and M. Khodabandeh, "A novel nonlinear hybrid controller design for an uncertain quadrotor with disturbances," Aerospace Science and Technology, vol. 45, pp. 294-308, 2015.

[9] T. Madani and A. Benallegue, "Backstepping sliding mode control applied to a miniature quadrotor flying robot," in IEEE Industrial Electronics, IECON 2006-32nd Annual Conference on, 2006, pp. 700705.

[10] T. Madani and A. Benallegue, "Sliding mode observer and backstepping control for a quadrotor unmanned aerial vehicles," in American Control Conference, 2007. ACC'07, 2007, pp. 5887-5892.

[11] H. Bouadi, M. Bouchoucha, and M. Tadjine, "Sliding mode control based on backstepping approach for an UAV type-quadrotor," World Academy of Science, Engineering and Technology, vol. 26, pp. 22-27, 2007.

[12] P. C. Garcia, R. Lozano, and A. E. Dzul, Modelling and control of mini-flying machines: Springer Science \& Business Media, 2006.

[13] M. K. Habib, W. G. A. Abdelaal, and M. S. Saad, "Dynamic modeling and control of a Quadrotor using linear and nonlinear approaches," 2014.

[14] G. M. Hoffmann, H. Huang, S. L. Waslander, and C. J. Tomlin, "Quadrotor helicopter flight dynamics and control: Theory and experiment," in Proc. of the AIAA Guidance, Navigation, and Control Conference, 2007.

[15] S. Bouabdallah, "Design and control of quadrotors with application to autonomous flying," École Polytechnique federale de Lausanne, 2007.

[16] R. Xu and Ü. Özgüner, "Sliding mode control of a class of underactuated systems," Automatica, vol. 44, pp. 233-241, 2008.

[17] F. Mehazzem, A. L. Nemmour, A. Reama, and H. Benalla, "Nonlinear integral backstepping control for induction motors," in Electrical Machines and Power Electronics and 2011 Electromotion Joint Conference (ACEMP), 2011 International Aegean Conference on, 2011, pp. 331-336.

[18] F. Kendoul, Z. Yu, and K. Nonami, "Guidance and nonlinear control system for autonomous flight of minirotorcraft unmanned aerial vehicles," Journal of Field Robotics, vol. 27, pp. 311-334, 2010. 


\section{BIOGRAPHIES OF AUTHORS}

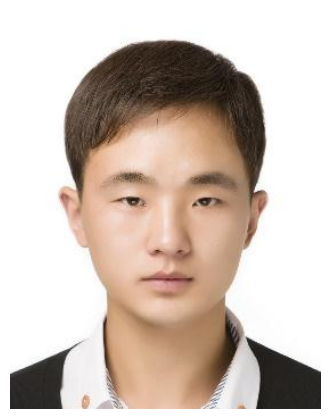

Since 2014, Chuan Lian Zhang is a master of electronic engineering school of Chonbuk National University. He graduated with honors in Mechanical Engineering from HUAIHAI Institute of Technology. His research area is about PLC automation control and waypoint navigation, trajectory tracking and obstacles avoidance of quadrotors.

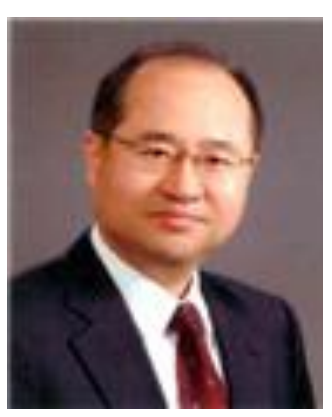

Currently, Kil To Chong is a professor and the dean of Electronics Engineering Division in Chonbuk National University, president of Electronics and IT New Technologies Research Center in Chonbuk National University. He received his BS degree from Oregon State University in Mechanical Engineering, got a master degree in Mechanical Engineering at Georgia Institute of Technology, US and a PhD in Mechanical Engineering at Texas A\&M University, US. He has published dozens of paers in international journals and confernnces. His interested fields are Time-Delay, Robotics, Artificial Intelligence and Sensor Networks. 